\title{
Counting Meromorphic Functions with Critical Points of Large Multiplicities
}

\author{
Dmitri Panov*, Dimitri Zvonkine ${ }^{\dagger}$
}

October 29, 2018

\begin{abstract}
We study the number of meromorphic functions on a Riemann surface with given critical values and prescribed multiplicities of critical points and values.

When the Riemann surface is $\mathbb{C} P^{1}$ and the function is a polynomial, we give an elementary way of finding this number.

In the general case, we show that, as the multiplicities of critical points tend to infinity, the asymptotic for the number of meromorphic functions is given by the volume of some space of graphs glued from circles. We express this volume as a matrix integral.
\end{abstract}

\section{From functions to constellations}

In this section we sketch the more or less standard construction that allows one to reduce the enumeration problem of meromorphic functions to a combinatorial problem of enumerating constellations. In the sequel we will be concerned with the latter problem. The construction is based on the Riemann existence theorem, exposed, for example, in [6].

Consider a compact connected Riemann surface $\Sigma$ and a non-constant meromorphic function $f$ on it. The function $f$ can be considered as a ramified covering of the Riemann sphere $\mathbb{C P}^{1}$ by the surface $\Sigma$. Denote by $w_{1}, \ldots, w_{k} \in \mathbb{C}$ the finite ramification points of the covering. (The point

*panov@ihes.fr, École Polytechnique, 91128 Palaiseau, France

†dimitri.zvonkine@math.u-psud.fr, bât 425, Université Paris-Sud, 91400 Orsay, France 
$\infty \in \mathbb{C P}^{1}$ might also be a ramification point.) Let $w_{0} \in \mathbb{C}$ be a finite noncritical value of $f$. Choose $k$ non-intersecting paths on the plane, connecting $w_{0}$ to the points $w_{1}, \ldots, w_{k}$. The cyclic order of the paths at the point $w_{0}$ should be equal to the order $w_{1}, \ldots, w_{k}$.

Definition 1 A star of loops on the complex plane is a set of $k$ non-intersecting closed loops $l_{1}, \ldots l_{k}$ of the following form. Each loop $l_{i}$ starts at $w_{0}$, follows the path from $w_{0}$ to $w_{i}$, goes around $w_{i}$ in the counterclockwise direction, and then goes back to $w_{0}$ following the path. A star of loops is shown in Figure 1.

In the sequel we suppose that the points $w_{0}, w_{1}, \ldots, w_{k}$ and the star of loops are fixed once and for all.

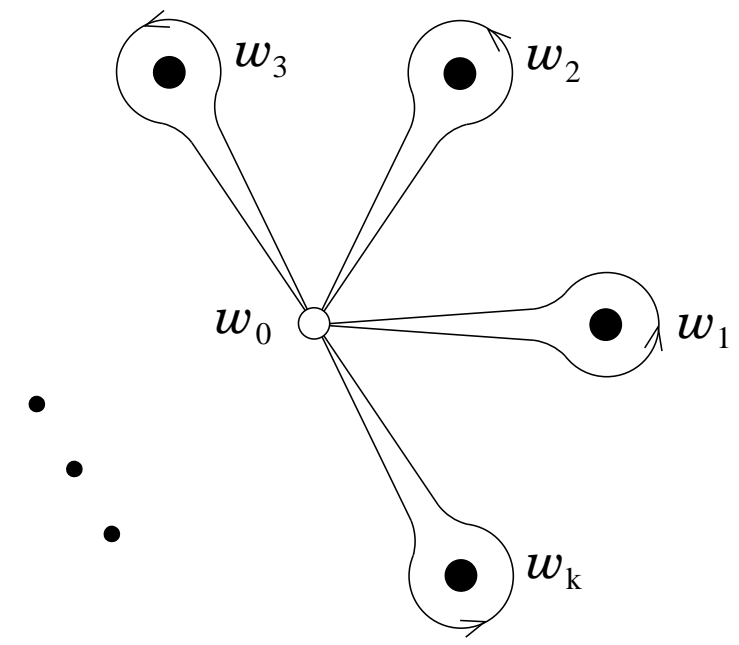

Figure 1: A star of loops.

The preimage under $f$ of the loop $l_{i}$ surrounding the critical value $w_{i}$ is composed of several curvilinear polygons on the Riemann surface $\Sigma$. The vertices of the polygon are the preimages of $w_{0}$. Its sides are the preimages of the loop $l_{i}$. Each polygon surrounds a unique point $z$ such that $f(z)=w_{i}$. If the polygon has more than one side, the corresponding point is critical; if it has only one side, it is not. Now we take the preimages of all the loops $l_{i}$ and erase all the 1-gons. We say that the polygons that compose the preimage of $l_{i}$ are of color $i$. 
Thus, on $\Sigma$, we obtain a graph consisting of polygons colored in $k$ colors, each polygon having at least 2 sides and surrounding exactly one critical point of $f$. The vertices of the polygons are the preimages of $w_{0}$. The polygons meeting at a vertex are all of different colors, and the cyclic order of the polygons meeting at a vertex is given by the increasing order of colors.

Definition 2 A $k$-constellation is a connected colored oriented graph consisting of oriented polygons (with at least 2 sides each) colored in $k$ colors (from 1 to $k$ ) and such that no two polygons of the same color share a common vertex.

A constellation can be uniquely (up to a homeomorphism) embedded into a (compact connected oriented) surface in such a way that (i) if we cut the surface along the constellation we obtain a union of domains homeomorphic to open discs, (ii) every polygon of the constellation surrounds one such disc and is oriented counterclockwise with respect to this disc, (iii) the cyclic order of polygons meeting at every vertex coincides with the increasing order of colors.

Definition 3 The embedding satisfying the conditions (i), (ii), and (iii) is called the natural embedding of a constellation.

It follows from Riemann's existence theorem that (once the star of loops is fixed) a constellation uniquely determines the couple $(\Sigma, f)$ up to a biholomorphic equivalence. The part of $\Sigma$ exterior to the polygons consists of several connected components corresponding to the poles of $f$. They will be called faces.

If $\Sigma$ is a sphere and $f$ a polynomial, the corresponding constellation is of a particular form, because $\infty$ is always a critical value of $f$ and its only preimage is $\infty$. One can easily see that the constellation is, in that case, a planar "tree" consisting of colored polygons. Such a constellation is called a cactus.

Definition 4 A $k$-cactus is a constellation that has no cycles other than the polygons it is glued from.

The surface of the natural embedding of a cactus is a sphere. Once a star of loops is fixed, a cactus determines the polynomial $f$ uniquely up to a change of variable $f(z) \mapsto f(a z+b)$. 
Thus the problem of enumerating meromorphic functions can be reduced to a combinatorial problem of enumerating cacti and constellations, and we will concentrate on the latter problem from now on.

\section{Spaces of gluings of circles}

The idea of this section is to replace by circles the polygons that compose cacti and constellations. The circles will be endowed with elements of length. This corresponds to letting the numbers of sides of the polygons tend to infinity. The number of cacti or constellations will be replaced by the volume of the space of possible gluings of circles. It turns out that these volumes are easier to compute then the numbers of cacti and constellations.

Definition 5 A circle constellation is an oriented colored graph with nonnegative lengths assigned to all edges, obtained by gluing together (at a finite number of points) several circles of given lengths colored in $k$ colors from 1 to $k$. Two circles of the same color cannot share a common point.

Definition 6 A circle cactus is a constellation of circles that has no cycles other than the circles themselves.

As above, a circle constellation (or a circle cactus) has a unique natural embedding into a compact surface (a sphere), satisfying the same three conditions as for ordinary constellations: (i) if we cut the surface along the circles we obtain a union of domains homeomorphic to open discs, (ii) each circle of the circle constellation surrounds one such disc and is oriented counterclockwise with respect to it, (iii) the cyclic order of circles at every vertex coincides with the increasing order of colors.

The connected components of the part of the surface exterior to the circles are called faces.

Suppose a set of $m$ oriented colored circles with fixed lengths is given. Let us fix a genus $g$ and a number of faces $p$. We can then consider the space of all possible gluings of the circles that give a circle constellation with $p$ faces on a surface of genus $g$.

Proposition 7 The set of circle constellations of genus $g$ with $p$ faces glued of a given set of $m$ circles has a natural structure of a singular smooth noncompact manifold of dimension $d=4 g-4+m+2 p$ with a volume measure. 
Proof. First we will describe the space of circle constellations with numbered vertices. Such constellations do not have nontrivial automorphisms, which simplifies the description.

Let us describe the smooth part of the space of circle constellations. A circle constellation belongs to the smooth part if all its circles intersect only two by two. In other words, three circles never share a common vertex. Let $\lambda_{1}, \ldots, \lambda_{q}$ be the lengths of the arcs into which one of the circles is divided by the vertices. The sum of these lengths is equal to the length of the circle (which is fixed), but, for example, the first $q-1$ lengths can take arbitrary values in an open domain in $\mathbb{R}^{q-1}$. Thus, taking for each circle the lengths of all its arcs except one, we obtain a set of local coordinates of the space of circle constellations. It is easy to check that the total number of these arcs is indeed equal to $d$.

Now consider a smooth family of circle constellations depending on a parameter $t$, such that, as $t$ tends to 0 , the lengths of some of the arcs tend to 0 . Then the gluing that we obtain in the limit is still a gluing of the same circles embedded into a surface of genus $g$ with $n$ faces, but the cyclic order of the circles at the vertices is not necessarily the same as the increasing order of the colors. If the cyclic order of the circles at each vertex happens to coincide with the increasing order of colors, then what we have obtained is a true circle constellation. It is the limit of the above family and lies in the nonsmooth part of the space of gluings. If, on the contrary, the cyclic order of circles at at least one vertex differs from the increasing order of colors, then the above family has no limit. This accounts for the noncompactness of the space of gluings.

Now we can introduce the volume measure. Consider the lengths of arcs $\lambda_{i}, 1 \leq i \leq d$ that form a set of local coordinates on the smooth part of the space of gluings. Consider the differential form $d \lambda_{1} \wedge \ldots \wedge d \lambda_{d}$. If we change the numbering of the arcs, this differential form will either change its sign or remain unchanged. Thus its absolute value is a well-defined volume measure on the space of gluings. We let the measure of the nonsmooth part be equal to 0 .

If we now consider the space of circle constellations whose vertices are not numbered, it is obtained from the above space of circle constellations with numbered vertices by factoring by a finite group action (the renumbering of the vertices). Therefore it is also a smooth manifold with singularities. The volume measure being indepenent of the numbering of the vertices, it descends to the factor space. $\diamond$ 
In the sequel we will also consider spaces of circle constellations with a fixed number of marked points on the circles. An obvious modification of the above construction shows that these spaces are also smooth manifolds with singularities and that they are endowed with a natural volume form. To see that, it suffices to cut every circle into arcs not only at the points where it is glued to other circles, but also at the marked points.

Now we are going to compare the number of constellations composed of a given set of polygons and the volume of the space of circle constellations composed of a given set of circles.

Suppose we are given a set of polygons colored in $k$ colors and a set of circles colored in $k$ colors. For each color, there is the same number of polygons as of circles. Denote by $n_{i j}$ be the number of sides of the $j$ th polygon of color $i$ and by $l_{i j}$ the length of the $j$ th circle of color $i$. We assume that polygons of the same color and number of sides are labeled, so that all the polygons are distinguishable. Similarly, the circles of the same color and lengths are labeled, so that all the circles are distinguishable.

Consider all constellations and circle constellations of genus $g$ with $p$ faces. Denote by $P\left(n_{i j}\right)$ (respectively $Q\left(l_{i j}\right)$ ) the number of constellations (respectively the volume of the space of circle constellations) like that composed of the above set of polygons (circles). Finally, let $m$ be the total number of polygons (circles) and $d=4 g-4+m+2 p$.

Theorem $8 P$ is a polynomial in the variables $n_{i j}$ of degree $d . Q$ is a homogeneous polynomial in the variables $l_{i j}$ of degree $d$. Moreover, $Q$ is obtained by taking the top degree homogeneous part of $P$ and substituting $l_{i j}$ for $n_{i j}$. In particular, if we substitutes $n_{i j}$ for $L_{i j}$ in $Q\left(l_{i j}\right)$, we obtain an asymptotic of the numbers $P\left(n_{i j}\right)$ for large $n_{i j}$.

Proof. Suppose a constellation is given. We call the topological type of the constellation the following information. First, for each polygon we retain the number of vertices at which it is glued to some other polygons. Second, enumerating these vertices in the cyclic order in which they appear on the polygon, we list, for each vertex, the polygons that are glued to it. The information that we do not retain is the number of edges that lie between the listed vertices.

It is easy to see that the number of constellations of a given topological type is a polynomial in the $n_{i j}$. Indeed, suppose that the $j$ th polygon of color 
$i$ is glued to other polygons at $k_{i j}$ vertices. Then there are

$$
\left(\begin{array}{l}
n_{i j}-1 \\
k_{i j}-1
\end{array}\right)
$$

ways to choose these vertices on the polygon. The total number of constellations of a given topological type is equal to the product of the above numbers over all the polygons (because the choices of the vertices are independant for different polygons). The top degree terms are obtained from the topological types in which no 3 polygons share a common vertex.

A topological type of a circle constellation is defined in exactly the same way as for constellations: for each circle we list the points at which it is glued to other circles and the circles that are glued at each of these points; the lengths of arcs between these points are not retained.

If in a topological type at least 3 circles share a common point at least once, than such a type determines a sub-manifold of the space of gluings of positive codimension. Therefore its contribution to the total volume is 0 . If, on the other hand, no 3 circles share a common point, the topological type determines a component of the space of gluings with nonzero volume. As above, denote by $k_{i j}$ the number of points on the $j$ th circle of color $i$ at which it is glued to other circles. The volume of the space of choices of these points equals

$$
\frac{1}{\left(k_{i j}-1\right) !} l_{i j}^{k_{i j}-1} .
$$

The volume of the whole component determined by the topological type is the product of these numbers over all circles.

Since taking the top degree term of

$$
\left(\begin{array}{l}
n_{i j}-1 \\
k_{i j}-1
\end{array}\right)
$$

and replacing $n_{i j}$ by $l_{i j}$ gives us precisely

$$
\frac{1}{\left(k_{i j}-1\right) !} l_{i j}^{k_{i j}-1},
$$

the theorem follows. $\diamond$

Example 9 Suppose we are given 3 polygons (circles) of different colors and with numbers of sides $n_{1}, n_{2}, n_{3}$ (respectively with lengths $l_{1}, l_{2}, l_{3}$ ). Then we have $P=n_{1}+n_{2}+n_{3}-2, Q=l_{1}+l_{2}+l_{3}$ (see Examples 11 and 15). 
Theorem 10 The volume of the space of circle cacti glued from $k$ circles of pairwise distinct colors and with lengths $l_{1}, \ldots, l_{k}$ is equal to

$$
\left(l_{1}+\ldots+l_{k}\right)^{k-2} .
$$

Example 11 Let $k=3$. Then the three circles are necessarily glued together in a chain. Suppose the circle number 1 is in the middle (Fig. 2).

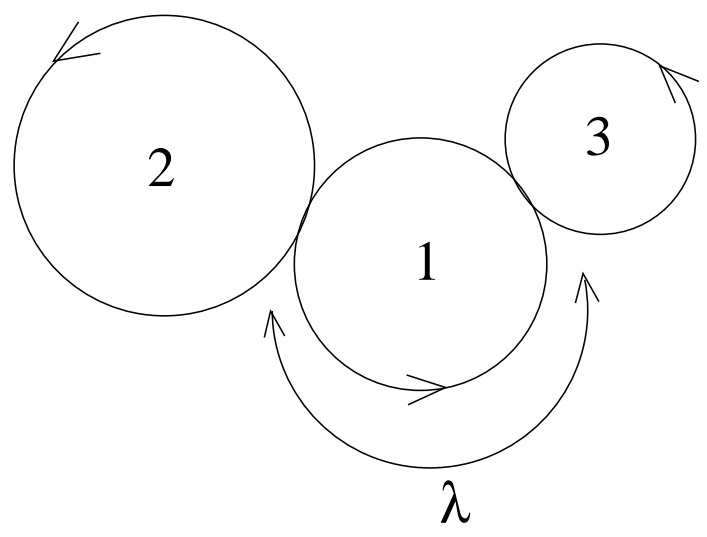

Figure 2: A circle cactus with 3 circles.

Then the length $\lambda$ of the arc between the points at which it is glued to the circles 2 and 3 can vary from 0 to $l_{1}$. Thus the component of the space of gluings that corresponds to the first circle being in the middle has volume $l_{1}$. Similarly, there are two more components with volumes $l_{2}$ and $l_{3}$. The total volume of the space of circle cacti is equal to $l_{1}+l_{2}+l_{3}$.

Proof of Theorem 10. We are going to describe a volume-preserving tranformation that allows one to reduce by 1 the number of circles (and of colors) by merging two circles into one and adding their lengths. On the new circle cactus thus obtained one point will be marked, so that the initial circle cactus can be recovered from the new one. After applying this merging procedure $k-1$ times, we will obtain a unique circle of length $l_{1}+\ldots+l_{k}$ with $k-1$ marked points. The space of possible markings will thus have the same volume as the space of circle cacti, and this volume is equal to

$$
\left(l_{1}+\ldots+l_{k}\right)^{k-2} .
$$


We start by making a list of the lengths $l_{1}, \ldots, l_{k}$. They will be needed to reverse the merging procedure.

Suppose we want to merge the circle of color 1 with the circle of color $i$. This merging is shown in Figure 3 .

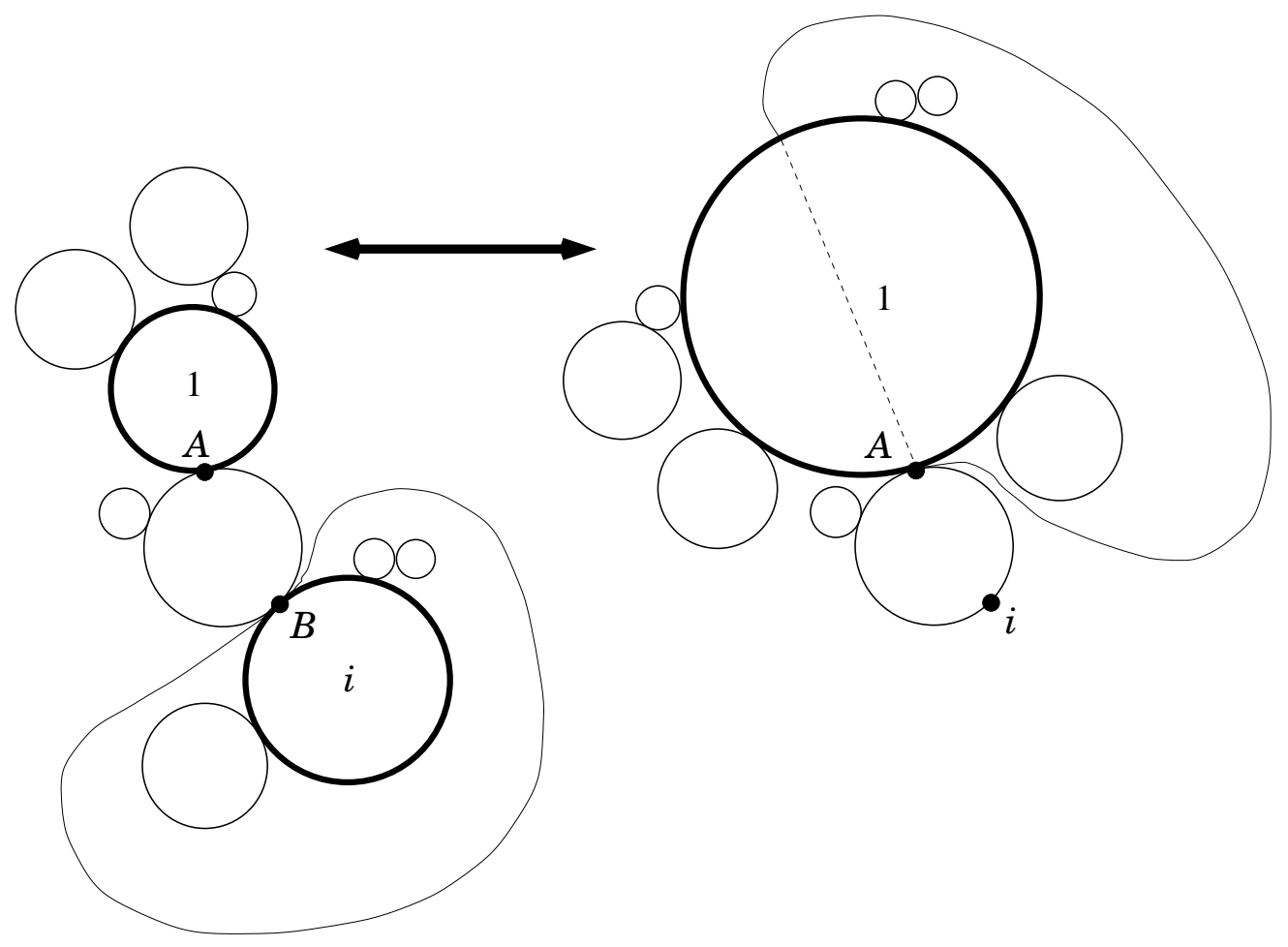

Figure 3: Merging two circles.

Since the circle cactus has no cycles, there exists a unique path of circles leading from the 1 st to the $i$ th circle. Denote by $A$ and $B$ the points of the 1 st and $i$ th circles respectively, at which this path begins and ends. Now we cut our circle cactus in two at the vertex $B$. The $i$ th circle and all circles attached to it belong to one part, while the other circles belong to the other part. This cutting is not well defined when more than two circles meet at the point $B$, but the subspace of such circle cacti has zero volume, so it does not matter for us. We then take the part containing the $i$ th circle and "open" it at the point $B$ (so that the $i$ th circle is transformed into an oriented arc). Then we similarly open the 1 st circle at the point $A$, obtaining another 
oriented arc. The path that leads from $A$ to $B$ remains attached to the end (and not the beginning) of this arc. Finally, we glue the two arcs together at their endpoints. The circles 1 and $i$ have merged into a single circle. We mark by the number $i$ the point that was called $B$ before.

It is easy to see that this operation can be reversed without ambiguity (except on the zero-volume set, where the point $B$ is a common point of two or more circles). During the inverse operation the circle of color 1 is cut in two arcs of lengths $l_{1}$ and $l_{i}$. Then the arcs are closed to form two circles, and, finally, one of them is moved to the marked point labeled by $i$.

Performing this merging procedure for all $i$ from 2 to $k$ we end up with a single circle of length $l_{1}+\ldots+l_{k}$ with $k-1$ points marked by numbers from 2 to $k$. From this marking we can recover the initial circle cactus, so it can be considered as an encoding of the circle cactus.

Thus the volume of the space of circle cacti is equal to

$$
\left(l_{1}+\ldots+l_{k}\right)^{k-2} \text {. }
$$

$\diamond$

As a by-product of our considerations we obtain a new proof of the Cayley formula.

Corollary 12 The number of trees with $k$ numbered vertices is equal to $k^{k-2}$.

Proof. Let us replace every vertex of a tree by a circle of length 1 and glue circles together iff the corresponding vertices are joined by an edge. It is easy to see that to every tree with numbered vertices corresponds a component of the space of circle cacti whose volume is equal to 1 . Since the total volume of the space of circle cacti is $k^{k-2}$, there are $k^{k-2}$ components and thus $k^{k-2}$ trees. $\diamond$

Now we will find the volume of the space of circle cacti in the case when several circles may have the same color. Suppose we are given $k$ unordered lists of lengths (a list for each color). Denote by $l_{i}$ the sum of lengths of circles of color $i$, by $l$ the sum of lengths of all the circles, and by $m_{i}$ the number of circles of color $i$. Further, denote by $\mid$ Aut $_{i} \mid$ the number of permutations of the circles of color $i$ that preserve their lengths (for example, if among the circles of color $i 3$ have the same length and all the other lengths are different, then $\mid$ Aut $_{i} \mid=6$ ). 
Theorem 13 In the above notation, the volume of the space of circle cacti glued from the circles of given lengths is equal to

$$
l^{k-2} \prod_{i=1}^{k} \frac{\left(l-l_{i}\right)^{m_{i}-1}}{\left|\mathrm{Aut}_{i}\right|} .
$$

Proof. We are going to apply again the merging procedure shown in Figure 3, but this time to circles of the same color.

The first thing we do, is to make all the circles of the cactus distinguishable by labeling (in an arbitrary way) the circles that have the same color and length. The number of different labelings of the circles of color $i$ is equal to $\mid$ Aut $_{i} \mid$.

After fulfilling the merging procedure as many times as possible, we will obtain a circle cactus with only one circle of every color. Some points on the circles of this circle cactus will by marked by dots of different colors in such a way that a dot never lies on the circle of the same color.

The final circle cactus and the marking allow one to recover the initial circle cactus without ambiguity.

Finally, the volume of the space of markings of any circle cactus with only one circle of each color (and with given lengths of the circles) equals

$$
\prod_{i=1}^{k}\left(l-l_{i}\right)^{m_{i}-1},
$$

because the $m_{i}-1$ marked points of color $i$ lie on the circles of colors different from $i$, whose total length is $l-l_{i}$.

Thus the total volume of the space of circle cacti whose circles of the same color and length are labeled, is equal to the product of the volume $l^{k-2}$ (given by Theorem 10) and of the above volume of the space of markings. In order to obtain the volume of the space of circle cacti with circles without labeling, we divide this product by the number

$$
\prod_{i=1}^{k}\left|\mathrm{Aut}_{i}\right|
$$

of labelings. The final result is

$$
l^{k-2} \prod_{i=1}^{k} \frac{\left(l-l_{i}\right)^{m_{i}-1}}{\left|\mathrm{Aut}_{i}\right|} .
$$

$\diamond$ 


\section{Enumeration of cacti}

The two first theorems of this section were first proved by Goulden and Jackson (see [3]) using the Lagrange inversion theorem; then it was reproved by Lando and the second author by algebro-geometric methods (see [四]). Here we give elementary proofs. These proofs almost repeat the proofs of the two parallel theorems on circular cacti in the previous section (actually, the copy/paste procedure was used). The difference is that now we must be careful in counting the cases where several polygons are glued together at the same vertex, while in the case of circles such gluings formed a zero-volume set.

Theorem 14 Consider the cacti composed of $k$ polygons with, respectively, $n_{1}, \ldots, n_{k}$ sides, and whose colors are all different. Denote

$$
n=n_{1}+\ldots+n_{k}-k+1
$$

be the total number of vertices in such a cactus. Then the number of such cacti is equal to $n^{k-2}$.

Example 15 Suppose there are 3 polygons with $n_{1}, n_{2}$, and $n_{3}$ sides respectively. If the polygon number 1 is in the middle, the number $\nu$ of edges lying between the 2 nd and the 3 rd polygons can vary from 0 to $n_{1}-1$ (see Figure (1).

Thus there are $n_{1}$ possible cacti with the 1st polygon in the middle. Similarly, there are $n_{2}$ cacti with the 2 nd polygon in the middle and $n_{3}$ cacti with the 3rd polygon in the middle. But the cactus in which the three polygons share a common vertex has been counted three times. Thus the total number of cacti is $n_{1}+n_{2}+n_{3}-2=n$.

Proof of Theorem 14. We are going to describe a procedure of a re-gluing of the cactus that allows one to reduce by 1 the number of polygons (and of colors) by merging two polygons into one. On the new cactus thus obtained we will mark one vertex, so that the initial cactus can be recovered from the new one. After applying this merging procedure $k-1$ times, we will obtain a unique polygon with $n$ vertices, $k-1$ of which are marked. Such polygons will thus be in a one-to-one correspondence with the cacti, and their number is equal to $n^{k-2}$. 


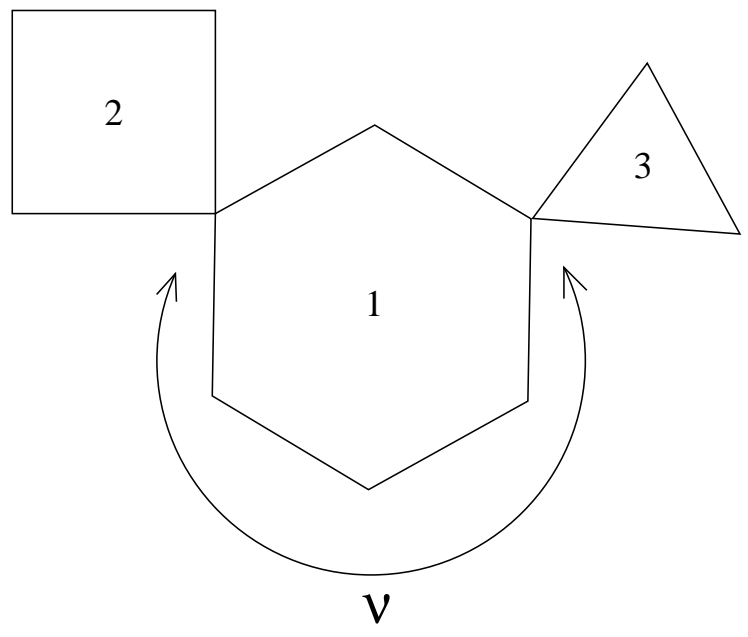

Figure 4: A cactus composed of 3 polygons.

First of all, we make a list of the numbers $n_{i}$ that will be needed to reverse the merging operation.

Let us describe how to merge the polygon of color 1 with the polygon of color $i$. This merging is shown in Figure 5 .

Consider the path of polygons leading from the 1st to the $i$ th polygon (it is unique because the cactus has no cycles). Denote by $A$ and $B$ the vertices of the 1 st and $i$ th polygons respectively, belonging to this path. Now we cut our cactus in two at the vertex $B$. The $i$ th polygon and all polygons attached to it at vertices different from $B$ belong to one part, while the other polygons belong to the other part. We then take the part containing the $i$ th polygon and "open" the vertex $B$ (so that the $i$ th polygon is transformed into a broken line). Then we erase the edge of the 1st polygon that follows the vertex $A$ in the counterclockwise direction (thus the 1st polygon is also transformed into a broken line). Finally, we glue the two broken lines together at their free ends. The polygons 1 and $i$ have merged into a single polygon. We mark by the number $i$ the vertex that was called $B$ before.

It is easy to see that this operation can be reversed without ambiguity and that it preserves the total number of vertices. When we reverse the operation, we cut the 1st polygon at one its vertices, so as to obtain a piece with $n_{1}-1$ edges and a piece with $n_{i}$ edges. The first piece is then closed with an additional edge, while the second piece is closed by gluing together 


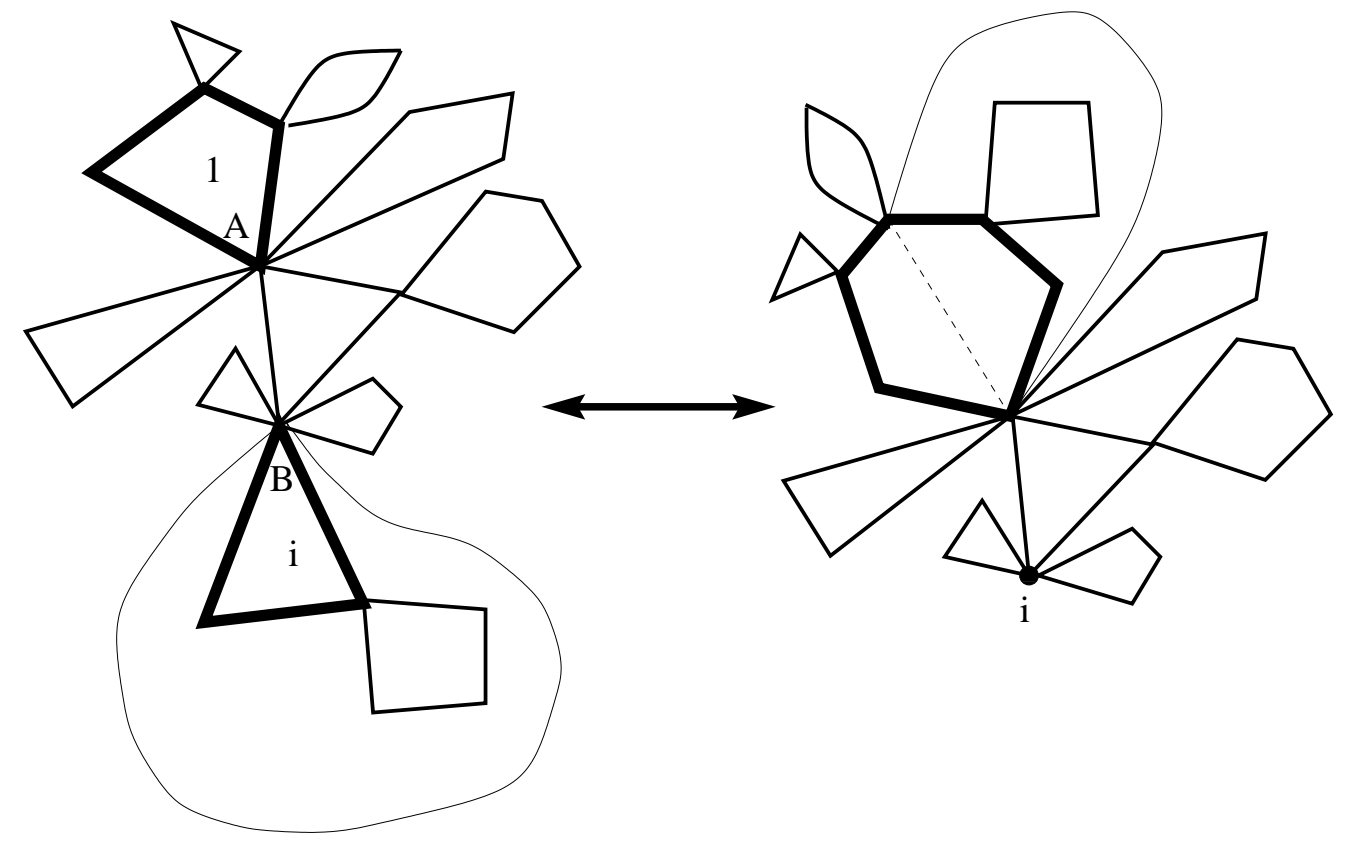

Figure 5: Merging two polygons.

its ends. The second polygon is then moved to the marked point with label $i$.

Performing this merging procedure for all $i$ from 2 to $k$ we end up with a single polygon with $n$ vertices, some of which are marked by numbers from 2 to $k$ (a vertex can be marked by several numbers simultaneously). From this marking we can recover the initial cactus, so it can be considered as an encoding of the cactus.

Thus the number of such cacti is $n^{k-2}$. $\diamond$

To a $k$-cactus we can assign a list $\mathbf{X}=\left(X_{1}, \ldots, X_{k}\right)$ of $k$ partitions of the number of its vertices. Their entries are all greater than or equal to 2 . The entries of the partition $X_{i}$ are simply the numbers of sides of the polygons colored in the color $i$.

Definition 16 The list $\mathbf{X}$ will be called the passport of the cactus.

Let $X$ be a partition whose all entries are greater than or equal to 2 and suppose that the entry 2 appears $a_{2}$ times, the entry 3 appears $a_{3}$ times, etc. 
Then we define the number of automorphisms of $X$ to be

$$
|\operatorname{Aut} X|=a_{2} ! a_{3} ! \ldots
$$

Cacti whose polygons are not necessarily of distinct colors may possess nontrivial symmetries. A symmetry of a cactus is a one-to-one map from the sets of its vertices and edges to themselves preserving adjacency and colors. Every cactus will be counted with the weight $1 /|\mathrm{Sym}|$, where $|\mathrm{Sym}|$ is its number of symmetries.

Theorem 17 Let $\mathbf{X}=\left(X_{1}, \ldots, X_{k}\right)$ be a passport. Denote by $p_{i}$ the number of entries of the partition $X_{i}$ and by $n_{i}$ the sum of these entries. Let $n$ be the total number of vertices of a cactus with passport $\mathbf{X}$.

Then the number of such cacti, each cactus being counted with the weight $1 / \mid$ Sym $\mid$, is equal to

$$
n^{k-2} \prod_{i=1}^{k} \frac{\left(n-n_{i}\right) !}{\left(n-n_{i}-p_{i}\right) !\left|\operatorname{Aut} X_{i}\right|} .
$$

Proof. We are going to apply again the merging procedure shown in Figure 5, but this time to polygons of the same color.

The first thing we do, is to make all polygons of the cactus distinguishable by labeling (in an arbitrary way) the polygons that have the same color and number of sides. The number of different labelings of the polygons of color $i$ is equal to $\left|\operatorname{Aut} X_{i}\right|$.

After fulfilling the merging procedure as many times as possible, we will obtain a cactus with only one polygon of every color. Some vertices of this cactus will by marked by dots of different colors and these markings will satisfy two conditions: first, a vertex marked by a dot of color $i$ does not belong to the polygon of color $i$; second, a vertex cannot be marked twice by dots of the same color (although it can be marked several times by dots of different colors).

The final cactus and the marking allow one to recover the initial cactus without ambiguity.

Finally, any cactus with only one polygon of each color (and with given numbers of sides of the polygons) has the same number of possible markings equal to

$$
\prod_{i=1}^{k} \frac{\left(n-n_{i}\right) !}{\left(n-n_{i}-p_{i}\right) !}
$$


On the other hand, the number of cacti whose all polygons are of different colors is equal to $n^{k-2}$ (Theorem 14). Multiplying this number by the number of markings, we obtain the number of cacti with passport $\mathbf{X}$ and with labeled polygons. In order to obtain the number of cacti with no labeling of polygons, we divide this product by the number

$$
\prod_{i=1}^{k}\left|\operatorname{Aut} X_{i}\right|
$$

of labelings.

We obtain that the number of cacti with passport $\mathbf{X}$ is equal to

$$
n^{k-2} \prod_{i=1}^{k} \frac{\left(n-n_{i}\right) !}{\left(n-n_{i}-p_{i}\right) !\left|\operatorname{Aut} X_{i}\right|}
$$

$\diamond$

Now we are going to consider the case of a rational function on the Riemann sphere with two poles: one of order $n$ and one simple. The case of rational functions with 2 poles was first studied by V. Arnold in [1]. If we put the poles at $\infty$ and 0 respectively, the function will take the form

$$
z^{n}+a_{1} z^{n-1}+\ldots+a_{0}+\frac{b}{z}
$$

(by a change $z \mapsto a z$ the leading coefficient can be chosen to be equal to 1 ). Counting the number of rational functions in this case is equivalent to finding the multiplicity of the Lyashko-Looijenga map on the strata of the $D_{n}$ singularity (see [5]). We will study only the case where each critical value has only one critical point (and several non-critical points) as its preimage. This corresponds to constellations with polygons of distinct colors. First, let us see what kind of constellations correspond to the rational functions of the above form.

Definition $18 \mathrm{~A}(1, n)$-constellation is a constellation with $n+1$ vertices satisfying the following conditions. Its natural embedding surface is a sphere. The exterior of the polygons of the constellation on the sphere consists of two cycles. In one of these cycles, called the small cycle, the colors of all the edges are distinct and their cyclic order is the inverse of the cyclic order of the colors. (The small cycle does not necessarily contain polygons of all colors.) 
Riemann's existence theorem implies that any $(1, n)$-constellation determines a rational function of the form

$$
z^{n}+a_{1} z^{n-1}+\ldots+a_{0}+\frac{b}{z}
$$

uniquely, up to a change of variables $z \mapsto \varepsilon z$, where $\varepsilon$ is an $n$th root of unity. The interior of the small cycle on the sphere contains the simple pole of the function.

Theorem 19 Suppose a set of $k$ polygons of distinct colors is given. The number of $(1, n)$-constellations glued from these polygons is equal to

$$
(k-1) n^{k-2} .
$$

Proof. First let us prove that the number of $(1, n)$-constellations of the above form is equal to

$$
\sum_{\substack{p \geq 2, q \geq 0 \\
p+q \leq k}} \sum\left(\begin{array}{c}
p+q \\
p
\end{array}\right)(p-1)^{q}(m-2 p-q)(n+1-p)^{k-p-q-1} .
$$

Here the second sum is taken over all choices of $p+q$ polygons among $k$, and $m$ is the total number of vertices in these polygons.

Indeed, the number $p$ is the number of polygons that form the small cycle. The number $q$ is the number of polygons that do not belong to the small cycle, but have a vertex lying on it. We are going to construct a $(1, n)$ constellation together with its embedding into the sphere. First we choose the numbers $p$ and $q$, then $p+q$ polygons among the $k$. Then we choose the $p$ polygons that will form the short cycle, which explains the factor $\left(\begin{array}{c}p+q \\ p\end{array}\right)$. There is a unique way to arrange these $p$ polygons into a cycle respecting the inverse cyclic order of colors.

Now we must glue each of the $q$ polygons to a vertex of the small cycle respecting the cyclic order of colors at each vertex. Since each of the $q$ polygons must remain in the exterior part of the short cycle, there is exactly one vertex of the short cycle where it cannot be glued. Once we have chosen the vertices to which the $q$ polygons will be glued, their cyclic order is automatically determined. Thus there are $(p-1)^{q}$ ways to glue the $q$ polygons. The 
obtained graph has $p$ vertices lying on the small cycle and $m-2 p-q$ "exterior" vertices. If, in the total $(1, n)$-constellation, we replace this subgraph by a unique polygon with $m-2 p-q$ vertices, we will obtain a cactus. Conversely, starting with a cactus containing a $(m-2 p-q)$-gon, we can replace this $(m-2 p-q)$-gon with the graph that we have constructed above and obtain a $(1, n)$-constellation. Therefore we just need to multiply the number of the above graphs by the number of cacti given by Theorem 14:

$$
(n-p+1)^{k-p-q-1} \text {. }
$$

This immediately gives the formula

$$
\sum_{\substack{p \geq 2, q \geq 0 \\
p+q \leq k}} \sum\left(\begin{array}{c}
p+q \\
p
\end{array}\right)(p-1)^{q}(m-2 p-q)(n-p+1)^{k-p-q-1} .
$$

Now note that the only factor in this formula that depends on the choice of the $p+q$ polygons (and not only on the numbers $p$ and $q$ ) is the factor $m-2 p-q$. Therefore we can rewrite the sum as follows:

$$
\sum_{\substack{p \geq 2, q \geq 0 \\
p+q \leq k}}\left(\begin{array}{c}
p+q \\
p
\end{array}\right)(p-1)^{q}(n-p+1)^{k-p-q-1} \sum(m-2 p-q) .
$$

Here the second sum is again taken over all the choices of $p+q$ polygons among the $k$, and $m$ is the total number of vertices in these polygons. The second sum can now be evaluated; it is equal to

$$
\left(\begin{array}{c}
k \\
p+q
\end{array}\right)\left[\frac{(n+k+1)(p+q)}{k}-2 p-q\right] .
$$

Indeed, the total number of vertices in all the polygons is equal to $n+k+1$. Therefore the average number of vertices in $p+q$ polygons is equal to

$$
\frac{(n+k+1)(p+q)}{k} \text {. }
$$

Substituting the value of the second sum in the initial expression, we obtain that the number of $(1, n)$-constellations is equal to

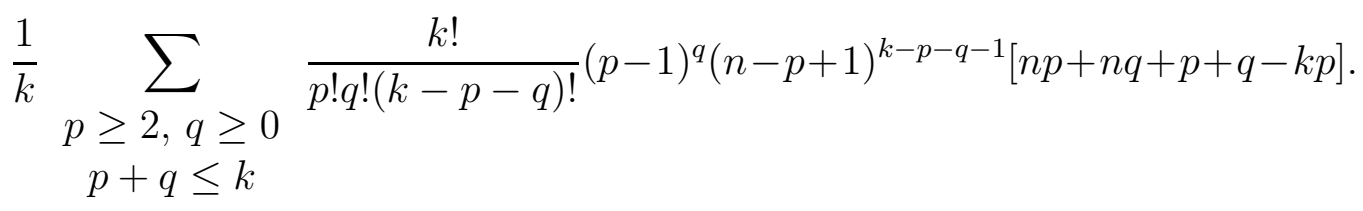


This expression depends only on $n$ and $k$, but not on the set of polygons that compose the $(1, n)$-constellation.

It can be evaluated by elementary but heavy computations. First we fix $p$ and carry out the summation over $q$. Using the formulas

$$
\sum\left(\begin{array}{c}
m \\
r
\end{array}\right) x^{r}=(1+x)^{m}
$$

and

$$
\sum\left(\begin{array}{c}
m \\
r
\end{array}\right) r x^{r}=m x(1+x)^{m},
$$

one gets

$$
\frac{1}{n k} \sum_{p=2}^{k}\left(\begin{array}{l}
k \\
p
\end{array}\right)[n p+p-k] n^{k-p} .
$$

Now, using once again the same two formulas, the summation can be carried out over $p$, and it gives

$$
(k-1) n^{k-2} .
$$

$\diamond$

\section{Matrix integrals}

In this section we show how to write a matrix integral that gives a generating function for the volumes of spaces of circle constellations glued of a given set of colored circles.

\subsection{Matrix models and the Wick formula}

First, let us briefly introduce the physical vocabulary concerning matrix models and one result that we will need: the Wick formula. For an accessible introduction to matrix models see [7]. Multi-matrix models are well explained in [2].

Let $E$ be a real vector space (in our case it will be the space of $k$-tuples of matrices of a special form). It is called the space of states of the model. To every point $x$ of $E$, we assign a nonnegative number $H(x)$, called the energy 
of $x$. (In our case $H$ will be a quadratic form on $E$.) The partition function of the model is defined by

$$
Z=\int_{E} e^{-\frac{1}{2} H(x)} d x .
$$

It can be thought of as the number of states of the model, each state counted with the weight $\exp \left(-\frac{1}{2} H\right)$. For any function $f$ defined on $E$, its expectation value is given by

$$
\langle f\rangle=\frac{1}{Z} \int_{E} f(x) e^{-\frac{1}{2} H(x)} d x .
$$

In the case when $H$ is a positive definite quadratic form on $E$, the Wick formula allows one to calculate the expectation values of polynomial functions on $E$. Denote by $H^{-1}$ the symmetric bilinear form, dual to $H$, on the dual vector space $E^{*}$. (If we choose a basis of $E$ and the dual basis of $E^{*}$, then the matrix of $H^{-1}$ is the inverse of the matrix of $H$.) Let $\lambda_{1}, \ldots, \lambda_{n} \in E^{*}$ be $n$ linear forms on $E$, and $f=\lambda_{1} \ldots \lambda_{n}$ their product. Then the expectation value of $f$ equals 0 if $n$ is odd and, if $n$ is even, it is given by the Wick formula:

$$
\langle f\rangle=\sum_{\text {parings of }\{1, \ldots, n\}} \prod_{\text {pairs }\{i, j\}} H^{-1}\left(\lambda_{i}, \lambda_{j}\right),
$$

where a pairing is a way to divide the indices $1, \ldots, n$ into $n / 2$ unordered pairs.

Let $E$ be the real vector space of $N \times N$ hermitian matrices and let the energy of a matrix $M$ equal $H(M)=N \operatorname{Tr} M^{2}$. Let

$$
f(M)=\frac{(N \operatorname{Tr} M / 1)^{k_{1}}}{k_{1} !} \frac{\left(N \operatorname{Tr} M^{2} / 2\right)^{k_{2}}}{k_{2} !} \ldots \frac{\left(N \operatorname{Tr} M^{p} / p\right)^{k_{p}}}{k_{p} !} .
$$

Then the Wick formula allows one to show that the expectation value of $f$ equals

$$
\sum \frac{1}{|\operatorname{Sym}|} N^{\chi}
$$

Here the sum is taken over all not necessarily connected embedded graphs with $k_{1}$ vertices of degree $1, k_{2}$ vertices of degree $2, \ldots, k_{p}$ vertices of degree $p$. $|\mathrm{Sym}|$ is the number of automorphisms of the graph, and $\chi$ is the Euler characteristic of the surface into which it is embedded.

The last result and the Wick formula are proved, for example, in [7]. 


\subsection{Expressing the volume of the space of gluings via matrix integrals}

Now we will show that the problem of computing the volume of the space of gluings of circles can be reduced to a matrix model with $k$ matrices.

We will therefore need a more elaborate version of the results reviewed in the previous subsection. This version is fit for colored graphs. The space $E$ will be the space of $k$-tuples of matrices $\left(A_{1}, \ldots, A_{k}\right)$ of a special form (a matrix per color). The quadratic form $H$ will encode the information that only vertices of distinct colors can be joined by an edge.

According to the Wick formula, the quadratic form that allows only vertices of different colors to be joined by edges satisfies

$$
H^{-1}=\sum_{i \neq j} \operatorname{Tr} A_{i} A_{j}
$$

The choice of the space $E$ is a consequence of this choice of $H$. More precisely, consider the complex vector space $E_{\mathbb{C}}$ of $k$-tuples of complex matrices $N \times N$. Then $E$ is its unique real subspace such that (i) $E \otimes_{\mathbb{R}} \mathbb{C}=E_{\mathbb{C}}$ and (ii) the restriction of $H$ on $E$ is a real positive definite quadratic form.

Suppose we are given a set of circles colored in $k$ colors and endowed with lengths. We suppose that the circles of the same color and length are labeled, so that all the circles are distinguishable. Denote by $l_{i j}$ the length of the $j$ th circle of color $i$. We are interested in the following generating function:

$$
F\left(N, l_{i j}\right)=\sum_{C} \operatorname{Vol}(C) N^{\chi(C)}
$$

Here the sum is taken over all topological types $C$ (see the proof of Theorem 8) of circle constellations that are not necessarily connected, but do not have isolated circles. $\operatorname{Vol}(C)$ and $\chi(C)$ are, respectively, the volume of the space of circle constellations of the given topological type, and the Euler characteristic of the (not necessarily connected) surface into which the constellation is embedded. Actually, we take into account only the topological types such that three circles never share a common point, because the contribution of the other topological types to the volume vanishes.

The generating function $F$ is a series in the variables $N$ and $l_{i j}$. The powers of $N$ that appear in $F$ are both positive and negative, but bounded from above. The powers of the $l_{i j}$ are positive. Suppose a term of $F$ contains 
$l_{i j}$ to the power $d-1$. We will soon see that it takes into account only the volume of those components of the space of circle constellations, on which the $j$ th circle of color $i$ touches $d$ other circles. In other words, looking at each term of $F$ we can say how many vertices each circle contains. Therefore we can also deduce the number of faces of the circle constellation.

We will express $F$ using a matrix model with the following space state $E$ and energy $H$.

Let $E$ be the space of $k$-tuples of matrices $A_{1}, \ldots A_{k}$ such that for all $i, A_{i}=X+Y_{i}$, where $X$ is a hermitian matrix and $Y_{i}$ are skew-hermitian matrices satisfying $\sum Y_{i}=0$. We identify the space $E$ with its dual $E^{*}$ using the symmetric nondegenerate (but not positive definite) bilinear form

$$
\left\langle\left(A_{1}, \ldots, A_{k}\right) \mid\left(B_{1}, \ldots, B_{k}\right)\right\rangle=\operatorname{Tr}\left(A_{1} B_{1}\right)+\ldots+\operatorname{Tr}\left(A_{k} B_{k}\right) .
$$

More generally, to any symmetric $k \times k$ matrix $S$ we can assign a bilinear form on $E$ given by

$$
\sum_{i, j=1}^{k} s_{i j} \operatorname{Tr}\left(A_{i} A_{j}\right),
$$

where $s_{i j}$ are the entries of $S$.

Denote by $I$ the unit $k \times k$ matrix and by $J$ the $k \times k$ matrix whose all entries are equal to 1 . The energy function $H$ on $E$ is given by the quadratic form associated to the matrix

$$
\frac{J-(k-1) I}{k-1}
$$

Thus the dual quadratic form $H^{-1}$ is given by the matrix $J-I$.

Proposition 20 Both quadratic forms $H$ and $H^{-1}$ are positive definite on $E$.

Proof. The quadratic form associated to the matrix $J$ is

$$
\begin{gathered}
\sum_{i, j} \operatorname{Tr}\left(X+Y_{i}\right)\left(X+Y_{j}\right)=k^{2} \operatorname{Tr} X^{2}+2 k \operatorname{Tr} X\left(Y_{1}+\ldots+Y_{k}\right)+\operatorname{Tr}\left(Y_{1}+\ldots+Y_{k}\right)^{2} \\
=k^{2} \operatorname{Tr} X^{2}
\end{gathered}
$$

because $Y_{1}+\ldots+Y_{k}=0$. 
The quadratic form associated to the matrix $I$ is

$$
\begin{aligned}
\sum_{i} \operatorname{Tr}\left(X+Y_{i}\right)^{2}= & k \operatorname{Tr} X^{2}+2 \operatorname{Tr} X\left(Y_{1}+\ldots+Y_{k}\right)+\sum_{i} \operatorname{Tr} Y_{i}^{2} \\
= & k \operatorname{Tr} X^{2}+\sum_{i} \operatorname{Tr} Y_{i}^{2} .
\end{aligned}
$$

Substituting these formulas in the expressions for $H$ and $H^{-1}$ we obtain

$$
H\left(A_{1}, \ldots, A_{k}\right)=\frac{1}{k-1}\left(k \operatorname{Tr} X^{2}-(k-1) \sum_{i} \operatorname{Tr} Y_{i}^{2}\right) ;
$$

and

$$
H^{-1}\left(A_{1}, \ldots, A_{k}\right)=k(k-1) \operatorname{Tr} X^{2}-\sum_{i} \operatorname{Tr} Y_{i}^{2} .
$$

Now, for a nonzero hermitian matrix $X$, we have $\operatorname{Tr} X^{2}>0$, while for a nonzero anti-hermitian matrix $Y$, we have $\operatorname{Tr} Y^{2}<0$. It follows that both $H$ and $H^{-1}$ are positive definite. $\diamond$

Now we give an expression of the function $F$ defined in the beginning of this subsection. Denote by $d \lambda$ a Lebesgue measure on the vector space $E$. (It is unique up to a scalar factor and we fix this factor once and for all.)

Theorem 21 The generating function $F$ is given by

$$
F\left(N, l_{i j}\right)=\frac{\int \prod_{i, j}\left(N \operatorname{Tr} \frac{e^{l_{i j} A_{i}}-1}{l_{i j}}\right) e^{-\frac{1}{2} N H} d \lambda}{\int e^{-\frac{1}{2} N H} d \lambda},
$$

where the integrals are taken over the space E.

More precisely, for any positive values of the $l_{i j}$ and for any positive integer $N$, both the integral above and the series $F$ converge and their values are equal.

Proof. First let us replace every constellation by the following dual picture (see Figure 6). Put a vertex in the center of each circle. Whenever two circles have a common point, draw an edge trough this common point, connecting the two corresponding vertices. Thus we obtain a graph embedded into the 
surface $\Sigma$. This opertion is well-defined whenever no three circles of the circle constellation share a common point, i.e., it is well-defined outside a set of measure 0 . The vertices of this graph are colored in $k$ colors, and a number $l_{i j}$ is assigned to each vertex. Each graph determines a component of the space of gluings of circles. Further, for a given gluing of circles, we can assign a number to each "angle" between two edges issued from the same vertex of the graph. This number is the length of the part of the circle that lies between the two corresponding gluing points.

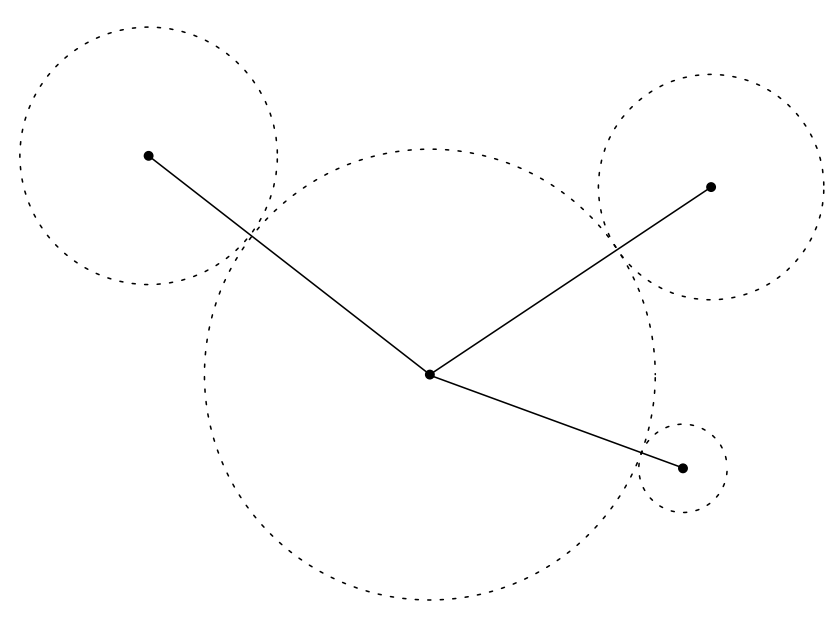

Figure 6: Replacing a constellation by its dual.

The sum of numbers surrounding a vertex is equal to the number $l_{i j}$ assigned to this vertex. If there are $d$ edges issued from this vertex, the number $l_{i j}$ must be divided into $d$ parts. The volume of the space of such divisions equals

$$
v_{i j}=\frac{l_{i j}^{d-1}}{(d-1) !} .
$$

If we consider a particular graph $G$, the volume of the corresponding component in the space of gluings of circles equals

$$
V_{G}=\prod_{\text {vertices of } G} v_{i j} .
$$

The volume of the total space of gluings is

$$
V=\sum_{G} V_{G} .
$$


Consider $k$ matrices $A_{1}, \ldots, A_{k}$ of size $N \times N$. Each matrix corresponds to vertices of a given color. Consider the quadratic form

$$
H^{-1}=\sum_{i \neq j} \operatorname{Tr}\left(A_{i} A_{j}\right)
$$

on the space of the $k$-tuples of matrices. The form $H^{-1}$ is chosen in such a way, that when we apply the Wick formula, it would tell us that we can join by edges any vertices of different colors, but not vertices of the same color.

Now we want to enumerate graphs with colored vertices and a number $l_{i j}$ attached to each vertex of color $i$. If the vertex is of degree $d$, it will be attributed a weight of

$$
\frac{l_{i j}^{d-1}}{(d-1) !} .
$$

Thus, in the matrix model, such a vertex corresponds to a term

$$
N \frac{l_{i j}^{d-1}}{(d-1) !} \frac{\operatorname{Tr} A_{i}^{d}}{d}
$$

Since the degree of each vertex can be arbitrary, and we want to take into account all graphs with all possible degrees of vertices, we must assign to a vertex of color $i$ and with number $l_{i j}$ the following sum

$$
N \sum_{d=1}^{\infty} \frac{l_{i j}^{d-1}}{(d-1) !} \frac{\operatorname{Tr} A_{i}^{d}}{d}=N \operatorname{Tr} \frac{e^{l_{i j} A_{i}}-1}{l_{i j}} .
$$

Multiplying such factors for all vertices and taking the expectation value of the product we obtain the integral from the statement of the theorem.

It remains to prove that the operation of taking the infinite sum

$$
\sum_{d=1}^{\infty} \frac{\operatorname{Tr}\left(l_{i j} A_{i}\right)^{d}}{d !}
$$

commutes with the integration. To prove that, note that all the partial finite sums

$$
\sum_{d=1}^{D} \frac{\operatorname{Tr}\left(l_{i j} A_{i}\right)^{d}}{d !}
$$


are bounded by the same function

$$
N e^{l_{i j}\left|A_{i}\right|}
$$

where $\left|A_{i}\right|$ is the sum of absolute values of the coefficients of the matrix $A_{i}$. This bound follows from the fact that the $N$ eigenvalues of $A_{i}$ are bounded by $\left|A_{i}\right|$. On the other hand, the product of a finite number of functions

$$
N e^{l_{i j}\left|A_{i}\right|}
$$

is integrable with respect to the measure

$$
e^{-N H} d \lambda
$$

because $H$ is a positive definite quadratic form, while $\left|A_{i}\right|$ increases only linearly with $A_{i}$. It follows that the integral in the statement of the theorem converges and is equal to the infinite sum of integrals that compute individual terms of the series $F$. Therefore the series $F$ itself converges to the same value as the integral. $\diamond$

\subsection{Computation of the integral}

We have been able to compute the integral of Theorem 21 only for $N=1$. Therefore the graphs corresponding to surfaces $\Sigma$ of different Euler characteristics are enumerated together.

More precisely, suppose as above that we are given a set of circles colored in $k$ colors and endowed with lengths $l_{i j}$. Denote by $f\left(l_{i j}\right)$ the generating function obtained from $F\left(N, l_{i j}\right)$ by setting $N=1$. If we fix the lengths $l_{i j}$ of the circles, the value of $f$ is the sum of volumes of the spaces of circle constellations on all compact surfaces. The constellations (and therefore the surfaces) are not necessarily connected, but do not contain isolated circles.

In the case $N=1$, the space $E$ is the space of $k$-tuples of complex numbers $\left(a_{1}, \ldots, a_{k}\right)$ of the form $a_{j}=x+i y_{j}$, where $x$ and $y_{j}$ are real

numbers, $\sum y_{j}=0$. The quadratic form $H^{-1}$ becomes

$$
H^{-1}=\sum_{i \neq j} a_{i} a_{j}
$$

\section{Theorem 22}

$$
f=\frac{1}{\prod_{i, j} l_{i j}} \sum_{\text {choices of } U_{i}, V_{i}}(-1)^{\left|V_{1}\right|+\ldots+\left|V_{k}\right|} e^{\frac{1}{2} H^{-1}\left(L_{1}, \ldots, L_{k}\right) .}
$$



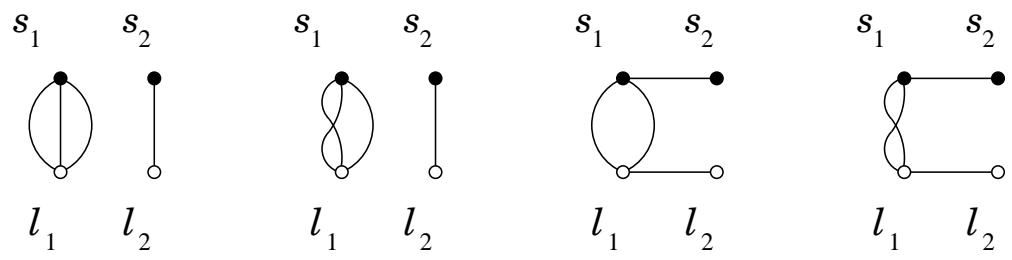

Figure 7: The four graphs that contribute to the term $\frac{2}{3} l_{1}^{2} s_{1}^{2}$.

Here the sum is taken over all possible ways to divide the circles of each color $i$ in two groups $U_{i}$ and $V_{i} .\left|V_{i}\right|$ is the number of circles in the group $V_{i} . L_{i}$ is the sum of lengths of circles in the group $U_{i}$.

Example 23 Suppose we have two colors and two circles of each color. The lengths of the circles of color 1 are $l_{1}, l_{2}$; those of the circles of color 2 are $s_{1}, s_{2}$. Then we have

$$
\begin{gathered}
\quad f=\frac{1}{l_{1} l_{2} s_{1} s_{2}}\left[e^{\left(l_{1}+l_{2}\right)\left(s_{1}+s_{2}\right)}-e^{\left(l_{1}+l_{2}\right) s_{1}}-e^{\left(l_{1}+l_{2}\right) s_{2}}-e^{l_{1}\left(s_{1}+s_{2}\right)}-e^{l_{2}\left(s_{1}+s_{2}\right)}+\right. \\
\left.+e^{l_{1} s_{1}}+e^{l_{1} s_{2}}+e^{l_{2} s_{1}}+e^{l_{2} s_{2}}-1\right]= \\
=2+\frac{3}{2}\left(l_{1} s_{1}+l_{1} s_{2}+l_{2} s_{1}+l_{2} s_{2}\right)+\quad \\
+\left[\frac{2}{3}\left(l_{1}^{2} s_{1}^{2}+l_{1}^{2} s_{2}^{2}+l_{2}^{2} s_{1}^{2}+l_{2}^{2} s_{2}^{2}\right)+\left(l_{1} l_{2} s_{1}^{2}+l_{1} l_{2} s_{2}^{2}+l_{1}^{2} s_{1} s_{2}+l_{2}^{2} s_{1} s_{2}\right)+\frac{3}{2} l_{1} l_{2} s_{1} s_{2}\right]+\ldots
\end{gathered}
$$

For instance, the term

$$
\frac{2}{3} l_{1}^{2} s_{1}^{2}
$$

corresponds to the four graphs in Figure 7 . In the figure we have drawn the graphs dual to the constellations, rather than the constellations themselves. They have two vertices of color 1 with valencies 1 and 3, and two vertices of color 2, also with valencies 1 and 3 .

Each of these graphs corresponds to a component of the space of constellations. For the two last graphs, the volumes of these components equal

$$
\frac{l_{1}^{2}}{2 !} \frac{s_{1}^{2}}{2 !} .
$$


The first two graphs have an additional symmetry group of order 3 , so their components have volume

$$
\frac{1}{3} \frac{l_{1}^{2}}{2 !} \frac{s_{1}^{2}}{2 !} .
$$

They add up to

$$
\frac{l_{1}^{2} s_{1}^{2}}{4}\left(1+1+\frac{1}{3}+\frac{1}{3}\right)=\frac{2}{3} l_{1}^{2} s_{1}^{2} .
$$

Proof of the theorem. Let us rewrite the integral to be computed for $N=1$ :

$$
f=\frac{\int \prod_{i, j} \frac{e^{l_{i j} a_{i}}-1}{l_{i j}} e^{-\frac{1}{2} H\left(a_{1}, \ldots, a_{k}\right)} d \lambda}{\int e^{-\frac{1}{2} H} H\left(a_{1}, \ldots, a_{k}\right) d \lambda} .
$$

Lemma 24 For any positive definite quadratic form $H$ and any linear form $\lambda$ on $E$, we have

$$
\frac{\int_{E} e^{-\frac{1}{2} H(x)+\lambda(x)} d x}{\int_{E} e^{-\frac{1}{2} H(x)} d x}=e^{\frac{1}{2} H^{-1}(\lambda)} .
$$

This is a reformulation of the fact that the Fourier transform of a Gaussian function is the Gaussian function corresponding the the dual quadratic form. $\diamond$

Consider the product

$$
\prod\left(e^{l_{i j} a_{i}}-1\right)
$$

in the numerator of the integral expressing $f$. Expanding this product we choose either the term

$$
e^{l_{i j} a_{i}}
$$

or the term -1 in each parenthesis. Thus for every $i$, the circles of colour $i$ are divided in two groups: we put a circle in the group $U_{i}$ if we have chosen

$$
e^{l_{i j} a_{i}}
$$

in the corresponding factor, and in the group $V_{i}$ if we have chosen -1 . Now, the product of any finite number of terms

$$
e^{l_{i j} a_{i}}
$$


is the exponent of a linear form on the space $E$. Therefore for each choice of terms we obtain an integral as in Lemma 24. Each of them can be calculated, and their sum is given in the statement of the theorem. $\diamond$

\section{Acknowledgments}

We are grateful to V. Arnold, Yu. Burman, M. Kazaryan, S. Lando, A. Zorich, and A. Zvonkin for useful and simulating discussions. We would also like to thank for their interest the members of Arnold's seminar in Moscow and of the mathematical seminars of Rennes University and of Moscow Steklov Institute.

\section{References}

[1] V. I. Arnold. Topological Classification of Trigonometric Polynomials and Combinatorics of Graphs with an Equal Number of Vertices and Edges, Functional Analysis and its Applications, vol. 30, No. 1, 1-17 (1996).

[2] P. Di Francesco. Matrix Model Combinatorics: Applications to Folding and Coloring. Random Matrices and their Applications, MSRI Publications, vol. 40, 111-170 (2001). http: //xxx. lanl.gov. Archive number: math-ph/9911002.

[3] I. P. Goulden, D. M. Jackson. The combinatorial relationship between trees, cacti and certain connection coefficients for the symmetric group, Europ. J. Combinatorics, vol. 13, 357-365 (1992).

[4] S. K. Lando, D. Zvonkine. On multiplicities of the LyashkoLooijenga mapping on the discriminant strata. Functional Analysis and its Applications, vol. 33, no. 3 (1999)

[5] E. Looijenga. The complement of the bifurcation variety of a simple singularity. Inventiones Mathematicae, vol. 23, 105-116 (1974).

[6] H. Völklein Groups as Galois Groups. An Introduction. Cambridge University Press, "Cambridge Studies in Advanced Mathematics", vol. 53 (1996).

[7] A. K. Zvonkin. Matrix integrals and map enumeration: An accessible introduction. Computers and Mathematics with Applications: Mathematical and Computer Modeling, special issue "Combinatorics and 
Physics" (M. Bousquet-Mélou, D. Loeb eds.), vol. 26, no. 8-10, 281304 (1997). 